www. revis tad y o. com

\title{
Influencias de los valores culturales en la mercadotecnia de servicios del automóvil
}

\author{
Influences of cultural values in the automotive marketing services
}

\author{
Alexander Fraß ${ }^{1 \text { y } 2}$, José Albors Garrigós ${ }^{1}$, Klaus-Peter Schoeneberg², Ángel Peiró Signes ${ }^{1}$ \\ 1 Universitat Politècnica de València \\ ${ }^{2}$ Hochschule für Angewandte Wisssenschaften Hamburgo, Berliner Tor 5, 20099, Germany \\ alexander.frass@haw-Hamburg.de, jalbors@omp.upv.es, klaus-peter.schoeneberg@haw-hamburg.de, anpeisig@omp.upv.es
}

Fecha de recepción: 26-11-2015

Fecha de aceptación: 22-02-2016

Resumen: Un factor importante en los servicios, en general, son los valores asociados a la cultura. Sin embargo, no existe suficiente literatura académica en este campo. Este trabajo enfoca estas limitaciones utilizando un modelo de valores culturales- el de Schwartz- para desarrollar un enfoque útil de instrumentación de estos valores. Estos se modelan como variables externas, para subrayar aquellos que son realmente relevantes. El trabajo se basa en una encuesta a clientes de talleres post venta en China de automóviles. Así, se evalúan los factores críticos de éxito, mediante modelos de ecuaciones estructurales de mínimos cuadrados parciales (PLS).

Palabras claves: Cultura, postventa, fidelización a la marca, PLS-SEM, teoría del valor de nivel Individual

\begin{abstract}
After-sales services have become very important in the automobile industry. However, this area has not been sufficiently researched, particularly with regard to China, the most important car market globally. In this respect, German manufacturers play a leading role because they dominate the premium market segment. When it comes to services, the one thing that is especially important in China is culture. At the same time, this is exactly where a scientific gap exists because the cultural aspect in automotive services has been mostly neglected in the research literature. Thus, specific knowledge with regard to Chinese service demand behaviour is lacking, which could become a crucial issue because of the enormous differences between Chinese and Western cultures. This paper addresses this limitation by providing a guideline for how the entire process chain of after-sales services could be researched in China. In addition, it also introduces Schwartz's individual level value theory as a beneficial operationalisation approach to culture. Thereby, values are modelled as exogenous variables in order to show which ones are really causal. This significant advantage cannot be provided by national comparison studies, which are the ones that are most often conducted. A total of 301 Chinese workshop customers of Audi, BMW and Mercedes-Benz were surveyed in order to assess the critical success factors of after-sales services via partial least squares structural equation modelling.
\end{abstract}

Keywords: Culture, After-sales services, Brand loyalty, PLS-SEM, Individual level value theory.

\section{Introducción}

La industria del automóvil es una de las industrias más relevantes a nivel global. En consecuencia, existe amplia literatura académica sobre la misma. Sin embargo, no se han investigado suficientemente todos los aspectos de la cadena de valor y especialmente aquellas fases mas cercanas al consumidor. Tradicionalmente, la literatura académica ha estudiado temas como las ventas, el diseño de productos y otros aspectos operacionales. En este sentido, los fabricantes de automóviles (u Original Equipment Manufacturers: OEMs), son considerados como los principales agentes del sector (Diez \& Reindl, 2005). En general, el enfoque académico en la industria, ha migrado reciente- mente hacia los servicios - y además, la investigación en los servicios - asociados, se ha convertido cada vez en más substancial (Diez, 2009; Reichhuber, 2010; Saccani et al., 2006).

En este contexto, los servicios de postventa tienen un papel excepcional, puesto que - incluso a través de otras industrias - se consideran como actividades de alto margen de beneficio y valor añadido, incluso más rentable que el producto de base (Cavalieri et al, 2007; Goffin, 1999; Wise/Baumgartner, 1999). Por otra parte, diversos estudios apuntan como estos servicios acaban fomentando otros objetivos adicionales, como aumentar la satisfacción del cliente y la fidelidad a la marca (Hünecke, 2012; Saccani et al., 2006; Huber \& 
Herrmann, 2001), logrando ventajas competitivas y diferenciación para sus promotores (Goffin \& New, 2001; Asugman et al., 1997; Hull \& Cox, 1994) o tienen la capacidad de mejorar la imagen de marca (Cavalieri et al., 2007, Saccani et al., 2006, Gallagher et al., 2005). Por ello, se debe apuntar la necesidad de profundizar en la investigación en el área de posventa (Jönke, 2012; Gebauer et al., 2012). Mas aun, el hecho de que los estudios publicados se han llevado a cabo, sobre todo, en zonas de occidente de mercados saturados y maduros por lo que estos estudios sufren de cierto sesgo debido una autopercepción distorsionada.

Otra cuestión relevante, que se ha descuidado en la investigación sobre la comercialización del automóvil, es la influencia de la cultura y sus valores sobre el comportamiento de la demanda de servicios; a pesar de la escasez de publicaciones al respecto (Löffler \& Dekker, 2012; Wang, 2011; Gudergan, 2010; Zhang et al., 2008; Gong, 2003; Asugman et al., 1997). Zhang et al., (2008), específicamente sobre los estudios de la influencia de los valores culturales aplicada a los servicios de mercadotecnia. Así, este trabajo pretende "ir más allá de Hofstede", porque cuando se analiza la cultura prácticamente solo se han utilizado las dimensiones del modelo de Hofstede (1987). También se subraya la importancia de la relación cliente/servicio, que tiene lugar en los talleres post venta del automóvil.

Con objeto de enfocar los huecos de investigación mencionados, este manuscrito elabora un modelo innovador de investigación, que analiza la cadena de valor del proceso de servicio postventa de los fabricantes de automóviles y, en concreto, considerando los efectos culturales como determinantes causales. Para ello se ha basado en un estudio de campo (basado en consumidores) que se centró en marcas premium alemanas en un país emergente como China.

Se ha seleccionado China como campo de investigación debido a que, es el mercado líder para la industria alemana del automóvil a nivel, global (VDA, 2015). Las marcas alemanas poseen allí la segunda mayor cuota de mercado capturando alrededor del $80 \%$ del segmento de mercado premium (CAAM, 2013; Sha et al., 2013). Además de la importancia de su mercado, China posee una cultura Asiática colectivista, considerada totalmente diferente de las culturas o mercados occidentales, con segmentos de consumidores y patrones de comportamiento muy heterogéneos (Wang et al., 2012; Knörle, 2011; Köll, 2009; Xin-an et al., 2008; Cui \& Liu, 2001). Esta investigación se limita a consumidores urbanos chinos con experiencia en un taller de marca de fábrica de Audi, BMW o MercedesBenz.

\section{Marco contextual y teórico del estudio}

\subsection{Definiendo un indicador de éxito}

Esta investigación pretende estudiar las relaciones causa-efecto dentro del sector de la postventa de vehículos alemanes premium en China, analizando las influencias culturales y los efectos resultantes sobre el éxito de las empresas. Es por tanto importante definir lo que se entiende por éxito.

Dentro de los servicios de gestión de mercadotecnia y marca, se entiende que la fidelidad de marca es un objetivo básico (Esch, 2010), especialmente cuando se tarta de evaluar la satisfacción del cliente (Sivadas \& Backer-Prewitt, 2000). Knörle (2011) la investigado específicamente la fidelidad a la marca en China y opta por este indicador, para definir el éxito, porque la fidelidad a la marca genera ingresos estables (fomenta la recompra, crea un cambio emocional de barreras, los clientes se sienten menos atraídos por las alternativas de la competencia), fomenta el crecimiento de las ventas (aumenta la frecuencia, aumenta las recomendaciones de boca boca) y, finalmente, porque la fidelidad a la marca aumenta la rentabilidad.

Por ello, en este trabajo, se ha seleccionado la fidelidad a la marca como el indicador de éxito. Debe considerarse, por ello, que el mercado de automóviles premium en China es predominantemente un mercado de primeros compradores. Por esa razón, un enfoque de medición conductual basado en las recompras llevaría a un sesgo, ya excluiría una cantidad significativa de usuarios. Así, el trabajo se enfoca a los clientes de los servicios post venta, que también son potenciales compradores, y por lo tanto se mide la intención de recompra y recomendación.

\subsection{La cadena de valor y éxito del servi- cio de posventa en el automóvil}

Al considerar el sistema de objetivos de las empresas de servicio, Meffert \& Bruhn (2009) sostienen que el éxito depende de tres aspectos de los objetivos: aquellos enfocados a dimensiones de la empresa, aquellos orientados al cliente y los enfocados al personal. Debido al enfoque hacia el consumidor de este trabajo, el aspecto orientado al cliente es más relevante. Aquí, estos autores apuntan que este aspecto está directamente relacionado con determinantes de la conducta, razón por la cual puede considerarse como la clave que influye en los factores de servicio de post venta. 
Los objetivos relacionados con el pueden diferenciarse en tres grupos: psicológicos (e.g. calidad percepción y satisfacción del cliente), conductuales (e.g. comportamiento de recompra y recomendación) y económicos (por ejemplo, valor para el cliente). Estos tres objetivos están vinculados entre sí, como se expresa en la cadena de valor y éxito (ver Figura 1) propuesta por Meffert \& Bruhn (2009: 91).

La cadena de valor y éxito se puede definir en el sector de posventa del automóvil como se muestra en la Figura 1. Aquí se perciben los elementos claves del modelo de investigación propuesto con respecto a la calidad de los servicios post venta, la satisfacción del servicio post-venta, fidelidad al taller y fidelidad a la marca. Estos elementos se consideran significativos y vinculados entre si, como demuestran diversos estudios realizados en occidente y China (ver tabla 1).

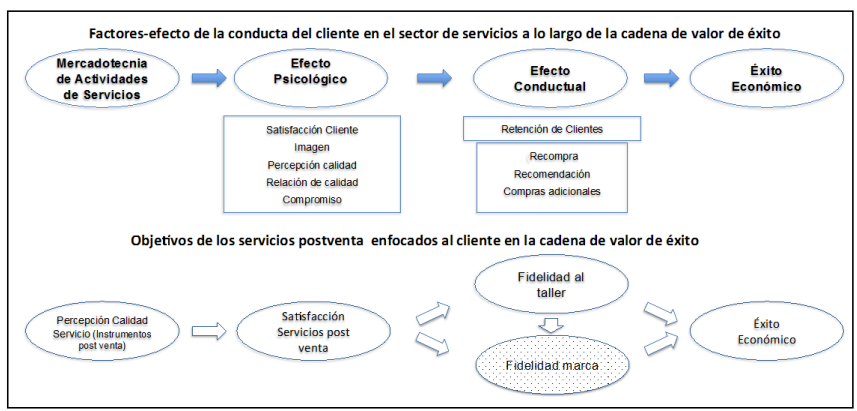

Figura 1. La cadena de valor y éxito en el sector de posventa del automóvil

(autores basado en Meffert \& Bruhn, 2009)

Además, se consideran tres constructos adicionales para incorporarlos al modelo de investigación. En primer lugar, la revisión de la literatura muestra gen- eralmente, que los costes de cambio son importantes, si se consideran las cuestiones relativas a la fidelidad (ver tabla 1). En segundo lugar, la imagen de marca se considera como crucial para los proveedores de servicios (Grönroos, 1984: 39 f.), ya que, por ejemplo, reduce la percepción del riesgo por parte del consumidor (Meffert \& Bruhn, 2009: 93 f.). En concreto, con relación al automóvil, la imagen de marca influye positivamente en las intenciones de compra, satisfacción con la marca y la futura compra o fidelidad (Esch \& Isenberg, 2013: 37 f.). Finalmente, la distancia aceptable al taller, expresada como la distancia máxima a la que un cliente esté dispuesto a llevar el automóvil a un taller determinado, se muestra a menudo como un factor importante (Diez, 2009: 301; Meffert \& Bruhn, 2009: 337; Lerchenmüller, 2007), pero casi no se ha investigado. Como resultado, estas tres variables se incorporan al modelo de investigación.

Como la literatura académica relativa a los constructos mencionados, relativos a la post venta, reconoce ampliamente, las relaciones de la cadena de valor de éxito, así como la contribución positiva de los constructos exógenos mencionados hacia la fidelidad de marca (ver tabla 1), se formulan como hipótesis. Sin embargo, además de la aceptación general en diversos sectores, estas relaciones realmente no han sido demostradas específicamente en el sector de postventa del automóvil. En este trabajo se supera esta limitación mediante una examen empírico de estas hipótesis, que se proponen del siguiente modo: "cuanto mayor sea el constructo exógeno, mayor será el endógeno"

Estas hipótesis, así como la literatura que los soporta, se muestran de forma detallada en la Tabla 1. Allí se detalla la bibliografía específica relevante en la posventa de automóviles.

Tabla 1: Resumen de las hipótesis

\begin{tabular}{|c|c|c|}
\hline \multicolumn{3}{|c|}{ Resumen de las hipótesis } \\
\hline No. & Hipótesis & Literatura relevante \\
\hline \multirow[t]{2}{*}{ H1 } & \multirow[t]{2}{*}{$\begin{array}{l}\text { Cuanto mayor sea la satisfacción de servicio post venta, } \\
\text { mayor será la fidelidad al taller. }\end{array}$} & $\begin{array}{l}\text { Hünecke, 2012: 127; Hättich, 2009: 213; Bei \& Chiao, 2001: 138; } \\
\text { Devaraj et al., 2001: } 434 \text { f.; Bloemer \& Pauwels, 1998: 82; Bloem- } \\
\text { er \& Lemmink, 1992: } 359\end{array}$ \\
\hline & & $\begin{array}{l}\text { Entrada adicional de las industrias de servicios en China: } \\
\text { Li et al., 2008: } 455 \text { f.; Deng et al., 2010: 295; Yim et al., 2008: } 751\end{array}$ \\
\hline H2 & $\begin{array}{l}\text { Cuanto mayor sea la fidelidad al taller, mayor sera la fi- } \\
\text { delidad a la marca. }\end{array}$ & $\begin{array}{l}\text { Hünecke, 2012: 128; Hättich, 2009: 213; Bloemer \& Lemmink, } \\
\text { 1992: } 359\end{array}$ \\
\hline
\end{tabular}




\begin{tabular}{|c|c|c|}
\hline \multirow[t]{2}{*}{ H3 } & \multirow[t]{2}{*}{$\begin{array}{l}\text { Cuanto mayor sea la calidad del servicio percibida, may- } \\
\text { or será la satisfacción de servicio post venta }\end{array}$} & $\begin{array}{l}\text { Hünecke, 2012: 147; Shuqin \& Gang, 2012: 177; Hättich, 2009: } \\
\text { 213; Bei \& Chiao: 2001: } 136 \text { ff.; Devaraj et al., 2001: } 434\end{array}$ \\
\hline & & $\begin{array}{l}\text { Entrada adicional de las industrias de servicios en China: } \\
\text { Deng et al., 2010: 296; Yim et al., 2008: } 751 .\end{array}$ \\
\hline $\mathrm{H} 4$ & $\begin{array}{l}\text { Cuanto mayor sea la percepción del coste de cambio de } \\
\text { taller, mayor sera la fidelidad al taller. }\end{array}$ & Peter, 1998: 77; Hättich, 2009: 213 ff. \\
\hline \multirow[t]{2}{*}{ H5 } & \multirow{2}{*}{$\begin{array}{l}\text { Cuanto mayor sea la percepción del coste de cambio de } \\
\text { taller mayor sea la fidelidad a la marca. }\end{array}$} & Verhoef et al., 2007: 108. \\
\hline & & $\begin{array}{l}\text { Entrada adicional de las industrias de servicios en China: } \\
\text { Deng et al., 2010: 295; Li et al., 2008: } 455 \mathrm{f} \text {. }\end{array}$ \\
\hline H6 & $\begin{array}{l}\text { Existe una relación entre la distancia aceptable al taller } \\
\text { y la satisfacción del servicio post venta o la fidelidad al } \\
\text { taller. }\end{array}$ & No hay disponibles estudios relacionados directamente. \\
\hline \multirow[t]{2}{*}{ H7 } & \multirow{2}{*}{$\begin{array}{l}\text { Cuanto mayor sea la imagen de marca, mayor será la } \\
\text { satisfacción del servicio post venta. }\end{array}$} & Devaraj et al., 2001: 434 \\
\hline & & $\begin{array}{l}\text { Entrada adicional de las industrias de servicios en China: Lai et } \\
\text { al., 2009: } 984\end{array}$ \\
\hline H8 & $\begin{array}{l}\text { Cuanto mayor sea la imagen de marca, mayor será la } \\
\text { fidelidad al taller. }\end{array}$ & Tu et al., 2014: $23 \mathrm{ff}$. \\
\hline \multirow[t]{2}{*}{ H9 } & \multirow{2}{*}{$\begin{array}{l}\text { Cuanto mayor sea la imagen de marca, mayor será la } \\
\text { fidelidad a la marca. }\end{array}$} & Hünecke, 2012: 128 \\
\hline & & $\begin{array}{l}\text { Entrada adicional de las industrias de servicios en China: Wang, } \\
\text { 2010: } 258 \text { f.; Ogba \& Tan, 2009: } 141\end{array}$ \\
\hline H10 & $\begin{array}{l}\text { La percepción de calidad del servicio post venta está in- } \\
\text { fluenciada significativamente por los valores culturales. }\end{array}$ & Knörle, 2011: 203 ff. \\
\hline $\begin{array}{l}\mathrm{H} 10 \\
\mathrm{a}-\mathrm{k}\end{array}$ & \multicolumn{2}{|c|}{$\begin{array}{l}\text { La percepción de la calidad del servicio post venta está influenciada significativamente por: conformidad (CON); tradición (TRA); } \\
\text { benevolencia (BEN); universalismo (UNI); uno mismo-dirección (SE-D); estimulación (STI); hedonismo (HED); logro (ACH); poder } \\
\text { (POW); seguridad (SEG). }\end{array}$} \\
\hline H11 & $\begin{array}{l}\text { La satisfacción del servicio post-venta está influenciada } \\
\text { significativamente por los valores culturales.. }\end{array}$ & $\begin{array}{l}\text { Van Birgelen et al., 2002: } 60 \text { f.; Reimann et al., 2008: 70; Frank et } \\
\text { al, 2013: } 2402 \mathrm{f} .\end{array}$ \\
\hline $\begin{array}{l}\mathrm{H} 11 \\
\mathrm{a}-\mathrm{k}\end{array}$ & \multicolumn{2}{|c|}{$\begin{array}{l}\text { La satisfacción del servicio post-venta está significativamente influenciada por: (CON); (TRA); (BEN); (UNI); (SE-D); (ITS); (HED); } \\
(\mathrm{ACH}) ;(\mathrm{POW}) ;(\mathrm{SEC}) \text {. }\end{array}$} \\
\hline K1Q & $\begin{array}{l}\text { La fidelidad a la marca está significativamente influenci- } \\
\text { ada por los valores culturales. }\end{array}$ & Lam, 2007: 15; Thompson et al., 2014: 2443; Yoo, 2008: 53 f. \\
\hline $\begin{array}{l}\text { K1Q } \\
\text { a-k }\end{array}$ & \multicolumn{2}{|c|}{$\begin{array}{l}\text { La fidelidad a la marca está significativamente influenciada por: (CON); (TRA); (BEN); (UNI); (SE-D); (ITS); (HED); (ACH); (POW); } \\
\text { (SEC). }\end{array}$} \\
\hline H13 & $\begin{array}{l}\text { La fidelidad al taller está influenciada significativamente } \\
\text { por los valores culturales. }\end{array}$ & No hay disponibles estudios relacionados directamente. \\
\hline $\begin{array}{l}\text { H13 } \\
\text { a-k }\end{array}$ & \multicolumn{2}{|c|}{$\begin{array}{l}\text { La fidelidad al taller está significativamente influenciada por: (CON); (TRA); (BEN); (UNI); (SE-D); (ITS); (HED); (ACH); (POW); } \\
\text { (SEC). }\end{array}$} \\
\hline
\end{tabular}




\subsection{La influencia causal de los valores culturales}

La cultura se considera, en este trabajo, como un flujo importante de influencias, que presumiblemente afecta al negocio completo de post-venta, o como Zhang et al. (2008) concluyen: cada dimensión de la experiencia de servicio al consumidor (expectativa, evaluación, reacción). Asimismo, los investigadores interculturales muestran cierto consenso, cuando argumentan que "la mayoría de los elementos del comportamiento del consumidor están ligados a la cultura" (De Mooij, 2014: 53) o que "la cultura, como una variable independiente significativa, determina el comportamiento del consumidor" (Emrich, 2007: 35).

Sin embargo, no existe una definición consistente de la cultura y existen varios métodos de enfoque y operacionalización. Como ya se ha mencionadó anteriormente, se aplican sobre todo las dimensiones de Hofstede (1987), pero el enfoque de este trabajo pretende ser más innovador. Por ello, se introduce el concepto de cultura en la figura 2 , ya que este enfoque conceptual de Osgood de 1951 ha sido aceptado ampliamente (Emrich, 2014; Meffert et al., 2010; Müller \& Gelbrich, 2004).

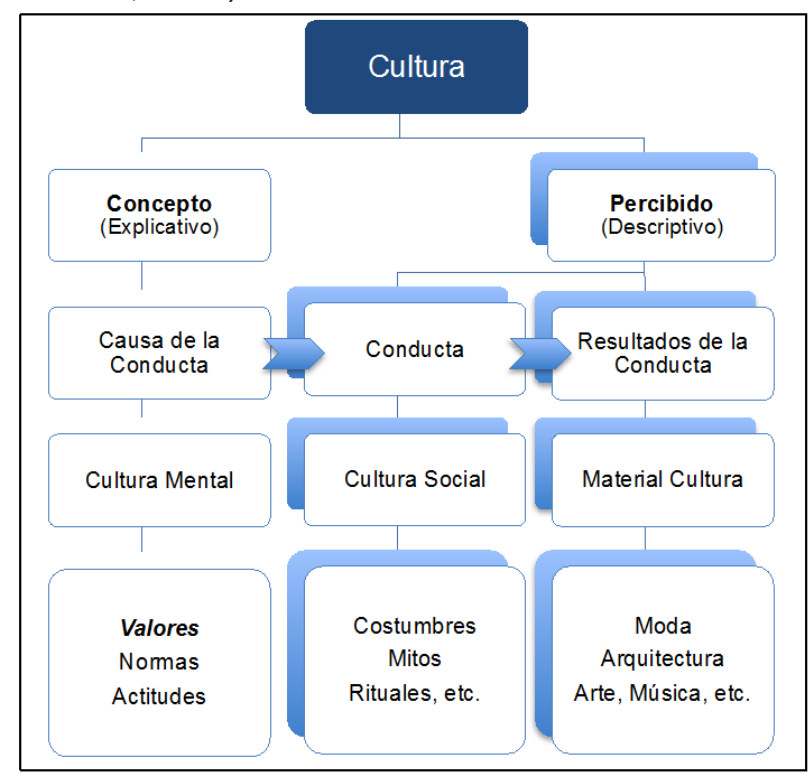

Figura 2. Concepto de cultura

(autores basado en Meffert \& Bruhn, 2010; Osgood, 1951).

Aquí, los valores culturales se describen como una manifestación relevante de la cultura de los individuos y el colectivo. Como representan las flechas, los valores o las actitudes y normas son causales al com- portamiento (Osgood, 1951) y por lo tanto suponen un anclaje posible a poner en práctica la cultura. La importancia de los valores es indudable, porque los valores son considerados como un elemento central de la cultura (Engelen \& Brettel, 2011; Schwartz, 2006; Müller \& Gelbrich, 2004; Hofstede, 1987) guiando las decisiones y las acciones del individuo (Srnka, 2005; Triandis, 1994; Schwartz, 1992).

Como los valores son causales, es posible utilizarlos como variables exógenas, lo que supone una aplicación poderosa para alcanzar el objetivo y explicar cómo la cultura afecta en China los servicios post venta.

Cuando estos valores tienen interés, la teoría de los valores individuales o básicos de Schwartz (1992) está más relacionada con nuestro objeto de investigación. $A$ esta se le considera como una de las teorías de valor teóricas más relevantes a nivel global (Cieciuch \& Davidov, 2012). Sin embargo, la inserción de Schwartz en la teoría psicológica podría ser la causa de su escasa aplicación en investigación de marketing (Zhang et al., 2008).

\subsection{Teoría de los valores nivel de Schwartz}

Schwartz define los valores básicos como..."metas trans-situacionales, que varían en importancia, y que sirven como principios en la vida de una persona o grupo." (Schwartz et al., 2012: 664; Schwartz, 1992: $1 \mathrm{ff.})$

Este autor identificó un conjunto amplio de diez valores a nivel individual, que son relevantes en cada sociedad. Los ordenó y organizó alrededor de un círculo motivacional como un sistema coherente. La estructura circular refleja una relación dinámica entre los valores, que puede expresarse como un conjunto de conflictos y congruencias, donde percibidos relativamente, los valores vecinos son similares en contraste con los valores dispuestos unos frente a otros (Davidov et al., 2008; Schwartz, 2003). Además, el constructo se puede consolidar como cuatro valores básicos de orden más alto en dos dimensiones ortogonales: Apertura al cambio versus Conservadurismo y Auto transcendencia versus Auto afirmación, como se muestra en la figura 3 (Davidov et al., 2008; Schwartz \& Boehnke, 2004). 


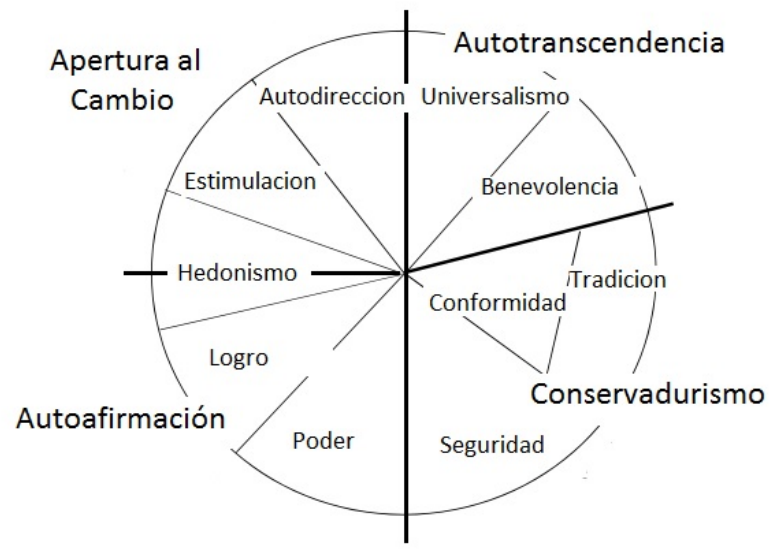

Figura 3: Relación estructural de los valores básicos (basado en Davidov et al., 2008)

En particular, esta teoría permite el análisis causal dentro de los países, a diferencia de los modelos de comparación nacional, utilizados hasta ahora (Hofstede, 1987), que sólo pueden mostrar las diferencia entre países, sin explicar la causalidad subyacente. Sobre este tema, diversos estudios han mostrado las influencias de las prioridades de valor individual sobre las actitudes y comportamientos como el activismo político o la actitud hacia el voto (Davidov et al., 2008: 441). Como resultado de los argumentos mencionados, los diez valores de nivel individuales se utilizan como constructos exógenos, porque esta metodología se adapta perfectamente al propósito de este trabajo.

Los diez valores motivacionales se presentan en detalle con la definición conceptual subyacente en la tabla 2 siguiente.

Tabla 2: Definición Conceptual de los valores básicos (basado en Schwartz, 1994).

\begin{tabular}{|l|l|}
\hline Valor & Definición conceptual \\
\hline Auto afirmación & $\begin{array}{l}\text { Pensamiento independiente y elección de } \\
\text { la acción, creando y explorando }\end{array}$ \\
\hline Estimulación & Emoción, novedad y reto en la vida \\
\hline Hedonismo & $\begin{array}{l}\text { Placer y gratificación sensual para uno } \\
\text { mismo. }\end{array}$ \\
\hline Logro & $\begin{array}{l}\text { Éxito personal a través de la demostración } \\
\text { de competencia según las normas social- } \\
\text { es }\end{array}$ \\
\hline Poder & $\begin{array}{l}\text { Estatus social y prestigio, control o domin- } \\
\text { io sobre otras personas y recursos }\end{array}$ \\
\hline
\end{tabular}

\begin{tabular}{|l|l|}
\hline Seguridad & $\begin{array}{l}\text { Seguridad, armonía y estabilidad de la so- } \\
\text { ciedad, delas relaciones y de uno mismo }\end{array}$ \\
\hline Conformidad & $\begin{array}{l}\text { Limitación de aquellas acciones, inclina- } \\
\text { ciones o impulsos que puedan molestar o } \\
\text { perjudicar a otros y violentar expectativas } \\
\text { o normas sociales }\end{array}$ \\
\hline Tradición & $\begin{array}{l}\text { Respeto, compromiso y aceptación de las } \\
\text { costumbres y las ideas que promueven la } \\
\text { cultura tradicional o la religión }\end{array}$ \\
\hline Benevolencia & $\begin{array}{l}\text { Preservación y mejora del bienestar de } \\
\text { las personas con quienes uno está en fre- } \\
\text { cuente contacto personal }\end{array}$ \\
\hline Universalismo & $\begin{array}{l}\text { Comprensión, apreciación, tolerancia y } \\
\text { protección hacia el bienestar de todas las } \\
\text { personas y la naturaleza }\end{array}$ \\
\hline
\end{tabular}

A fin de poner en práctica estos valores, existen diversos enfoques (véase por ejemplo Cieciuch \& Davidov, 2012). Por ello, se utiliza un enfoque PVQ (Portrait Values Questionnaire) con 21 cuestiones, ya que éste es extremadamente eficiente en tiempo y apropiado con respecto a los requisitos estadísticos de modelización de ecuaciones estructurales.

Zhang et al. (2008) han analizado en un artículo de revisión, con qué frecuencia se ha aplicado el enfoque cultural en la investigación de servicios. La teoría de Schwartz se ha aplicado únicamente una vez. Recientemente se han publicado algunos estudios, especialmente con un enfoque en China. Entre ellos, sólo Xiao \& Kim (2009) y Knörle (2011) se centran en el comportamiento del consumidor y ninguno se centra aspectos de satisfacción y fidelidad. Sin embargo, los constructos planteados que enfocan la percepción de la calidad del servicio, la satisfacción del servicio post-venta, la fidelidad al taller post venta y a la marca, muestran influencias culturales. Los estudios más significativos y específicos del contexto de Post venta se presentan en la tabla 1. Sin embargo, ninguno de estos trabajos ha utilizado la aplicación de los diez valores básicos de Schwartz en China con relación a la cadena de servicio postventa. Es por ello que el contraste de hipótesis de este trabajo superará esta limitación, enriqueciendo considerablemente el cuerpo de conocimiento y rellenando un hueco relevante en la investigación.

\subsection{Modelo de investigación}

La suma de constructos ya mencionados y sus relaciones, enunciados como hipótesis, se pueden or- 
ganizar en un modelo de relaciones estructuradas (ver figura 4).

Para presentar un modelo estructurado de forma clara, el constructo "Cultura1-4" se presenta como una agregación simbólica de cuatro sub constructos de cultura que tienen como objetivo una relación endógena con la cadena de valor de post venta: calidad de servicio percibida, Satisfacción del servicio post venta, fidelidad al taller post venta y fidelidad de marca.

Los cuatro sub constructos culturales son constructos auxiliares modelados como constructos formativos reflexivos de segundo orden.

Por qué hemos recurrido a este modelo? Si los valores de los diez factores (o dimensiones) culturales se modelaran independientemente, esto conduciría a una enorme complejidad y por lo tanto, afectaría negativamente la explicación estadística (Wetzels et al., 2009). Por ejemplo, el $\mathrm{R}^{2}$ aumenta en los modelos complejos, cuando muchos caminos apuntan hacia una constructo-objetivo (Hair et al., 2014). El enfoque de segundo orden utilizado simplifica el modelo sin carecer, necesariamente, de poder de explicativo ya que la influencia causal de cada valor del constructo individual se puede evaluar, con este método, mediante análisis de los efectos totales. Los constructos de segundo orden suponen un enfoque complejo con varios requisitos. Es un método que ha sido útil, en este trabajo, para explicar de las relaciones entre las variables (Hair et al, 2014b). Con objeto de de modelar constructos de segundo orden reflectivo-formativos, Becker et al. (2012) ofrecen una metodología particular, que se ha aplicado estrictamente en este caso.

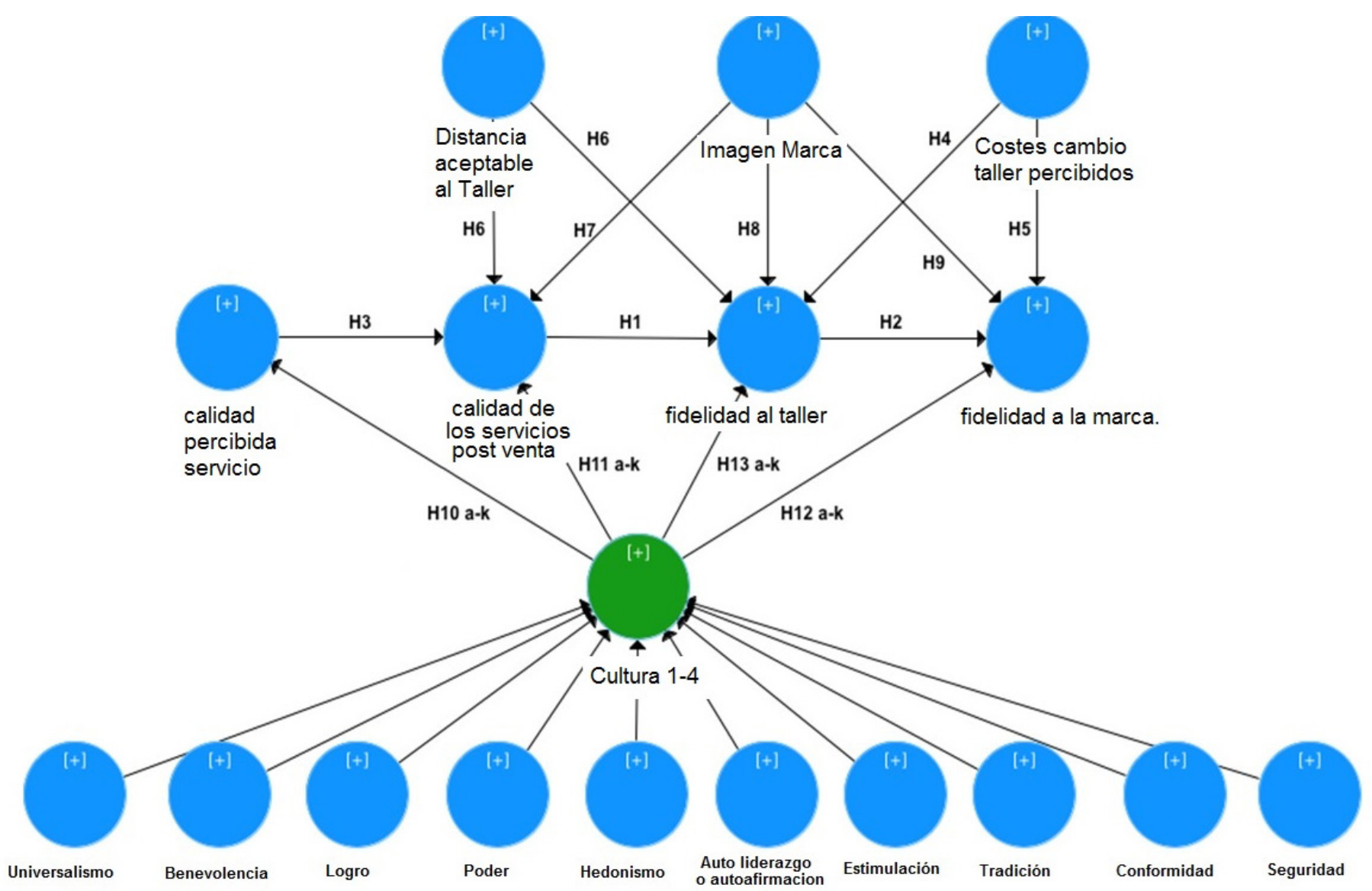

Figura 4. Modelo de investigación (autores) 


\section{Estudio empírico}

\subsection{Método de estudio y muestra}

Con objeto de recoger los datos necesarios para el análisis mediante mínimos cuadrados parciales (PLS) y modelarlos por medio de ecuaciones estructurales (SEM), se recurrió a una campaña de encuestas.

Para ello se desarrolló un cuestionario estandarizado que se distribuyó por medio de la web. Este contiene preguntas estructuradas según escalas Likert de calificación de uno a cinco puntos. Previamente, se realizaron dos análisis piloto previos a 30 sujetos con el cuestionario traducido al mandarín.

Posteriormente, se distribuyó la encuesta entre 434 consumidores chinos identificados como clientes de automóviles premium alemanes. De ellos 340 rellenaron la encuesta, resultando 330 encuestas completas.

Mediante un proceso de control de calidad se seleccionaron 301 encuestas válidas. Esta muestra se compone de $66 \%$ hombres y $34 \%$ mujeres, un $57 \%$ consumidores de Audi, un $29 \%$ de BMW y el $14 \%$ de Mercedes-Benz. Alrededor de un tercio vive en Shanghai, un $19 \%$ en Beijing $19 \%$ y un $19 \%$ en Guangdong. La variedad I de origen está asegurada con un $31 \%$ de encuestas procedentes de otros lugares. Conviene subrayar la ventaja de esta muestra ya que, en otros estudios en este área, los sujetos eran estudiantes de una sola zona geográfica (e.g. Knörle, 2012).

\subsection{Evaluación del modelo de investi- gación}

Con el fin de evaluar los modelos PLS-SEM se deben evaluar, en primer lugar, los modelos de medición externos. Aquí, es crucial distinguir entre constructos medidos reflexivos y formativos (Hair et al., 2014; Diamantopoulos \& Winkelhöfer, 2001) Posteriormente, se evalúa el modelo estructural. Si los resultados son aceptables, se puede considerar que el modelo es adecuado para realizar las experimentos de las hipótesis.

En este estudio, se cumplen todos los criterios de evaluación. Así, los modelos cumplen las pruebas de medición reflexiva, consistencia interna, validez convergente y validez discriminante. Igualmente ocurre con los modelos formativos de medición, la validez de contenido, la multicolinealidad entre indicadores y la importancia y relevancia del peso y las cargas exter- nas. Por último, se ha evaluado el modelo estructural - según las pruebas de multicolinealidad, $R^{2}, f^{2}, Q^{2} q^{2}$ que superan suficientemente todos los requisitos. Los resultados detallados se presentan en Apéndice 1-4.

\subsection{Resultados}

En esta sección se presentan los resultados de las pruebas de hipótesis. Así se muestra el contenido de cada prueba de hipótesis o relación. Los criterios detallados para aceptar o rechazar hipótesis se presentan en el Apéndice 1-4. Con respecto a las variables culturales, los efectos directos de los constructos de segundo orden, Cultura1-4, hacia los constructos endógenos se utilizan para comprobar las hipótesis $\mathrm{H} 10-\mathrm{H} 13$. La hipótesis posteriores relacionados a-k se comprueban a través de los efectos totales de los constructos de primer orden sobre los valores de nivel individuales. $\mathrm{Si}$, en esta estructura jerárquica, la relación principal es incapaz de satisfacer los criterios de prueba, se rechazan las hipótesis sub siguientes.

H1 La teoría postulada sobre la relación positiva entre la satisfacción del servicio post-venta y la fidelidad al taller de servicio se confirma empíricamente, muy significativamente, en un nivel $\alpha$ de 0.01 .

H2 que postula que cuanto mayor sea la fidelidad al taller, mayor sea la fidelidad a la marca se confirma también empíricamente, pero fuerza el coeficiente de relación $\square$ es un $43 \%$ menor que en la relación $\mathrm{H} 1$. Sin embargo, esta relación se considera relevante. Por otra parte, la fidelidad de taller es el facilitador más fuerte de la fidelidad de marca en el modelo.

H3 La relación entre calidad percibida del servicio postventa la satisfacción del servicio post venta tiene el coeficiente $\square \square$ más grande (0.673) de todo el modelo. En consecuencia $\mathrm{H} 3$ se valida.

Como la calidad del servicio es una variable formativa, la información adicional nos puede ayudar para explicar que son las variables más importantes que influyen en este constructo. Esta será particularmente relevantes, con respecto a las implicaciones de gestión, ya que cada elemento es comparable a los instrumentos de servicio post venta (del marketing mix) de los OEM's.

- $\quad$ El precio o los costes, una dimensión que a menudo se considera crucial, no es el moderador más importante sobre la calidad de servicio percibida en China. Ocupa unicamente la séptima posición sobre los nueve. 
- Los aspectos más importantes son, en primer lugar, la cercanía con un peso de 0,280, seguido del aspecto de las instalaciones $(0,234)$ y tercero la honestidad e integridad de la gerencia con un peso de 0,206).

- La profesionalidad para desarrollar el trabajo a tiempo es la dimensión menos importante, con un peso negativo pequeño-0,047 sobre la calidad percibida de servicio.

H4-H5 Los costes de cambio a un taller alternativo se han postulado para predecir la fidelidad de marca y taller, pero sólo la relación de fidelidad a la marca es significativa. Como resultado se rechaza la hipótesis H4 y se acepta la H5. En consecuencia, los costes de cambio de taller percibidos se pueden considerar un antecedente relevante de la fidelidad a la marca, ya que el valor del efecto $f^{2}$ es medio.

H6 Esta postulaba que existe una relación entre la distancia aceptable al taller y la satisfacción del servicio post venta o la fidelidad al taller Esta debe ser rechazada, porque ni existe una relación significativa, ni una contribución de $2 \mathrm{f}^{2} \mathrm{o} \mathrm{q}^{2}$. Este constructo no contribuye, en cualquier caso, al modelo de investigación.

H7 Aquí se confirma que la imagen de marca contribuye con un coeficiente $\beta$ significativo de 0,162 y un valor f2 pequeño sobre la satisfacción del servicio post venta; así H7 puede ser validada.

H8-H9 Ambas variables, con valores de relación $\beta$ similares $(0,162$ y 0,165$)$, son aceptables por lo que H8 y $\mathrm{H} 9$ se pueden validar; así la imagen de marca contribuye positivamente a la fidelidad de marca y taller.

En particular, la fidelidad a la marca como indicador de éxito, en la cadena de valor, es la variable con mayor $\mathrm{R}^{2}$ de todo el modelo, lo que nos indica que la fidelidad a la marca se puede explicar por variables exógenas con una varianza de $64,8 \%$.

H10 Dentro de los valores culturales relacionados con las hipótesis propuestas, la calidad de servicio percibida es el constructo más afectado. Esta relación es altamente significativa y se ha confirmado empíricamente.
El análisis detallado de los resultados relativos a las la hipótesis, H10a-k, muestra que la mayoría de los valores individuales no predicen un efecto significativo sobre la calidad de servicio percibida. Sin embargo, H10d es aceptada, por lo tanto el universalismo es un valor que resulta relevante $(\#=0,205)$ cuando influye sobre la percepción de la calidad del servicio post venta. Por otra parte, la auto afirmacion contribuye positivamente con un coeficiente de relación $\beta$ de 0,187 , pero sólo en un nivel $\alpha$ de 0,1 .

H11 La Satisfacción del servicio post-venta, no está influenciada significativamente por cualquier factor individual de los valores culturales, por lo que la hipótesis principal y las subsecuentes hipótesis (H11 a-k) deben ser rechazadas.

H12 La fidelidad a la marca está significativamente influenciada por los valores culturales, razón por la cual se acepta H12. A nivel individual, la auto afirmacion resulta el valor más importante, con un coeficiente $\beta$ de 0,136 . Igualmente, el universalismo tiene cierta relevancia (con un $\beta$ de 0,101 ), pero con un nivel $\alpha$ de significación de 0,1.

Las hipótesis restantes, $\mathrm{H} 12$ a-c y $\mathrm{H} 12$ f-k, son rechazadas, al no presentar soporte empírico.

H13 Finalmente, la fidelidad al taller- como el principal facilitador del indicador de éxito de fidelidad a la marca, puede predecirse significativamente por solo un valor individual cultural- el universalismo (con una $\#=0,158)$, por lo tanto se aceptan H13 y H13d. En consecuencia, las restantes hipótesis secundarias son rechazadas, debido a su insignificancia.

Por último, Figura 5 resume las relaciones que pueden ser aceptadas empíricamente. El peso de cada relación (coeficiente $\square$ ) se indica por el grosor de la flecha, y el nivel de significancia se muestra a través de asteriscos. Los efectos totales de los valores de cultura individuales se simbolizan en la figura como contribuyentes de segundo orden. El número de hipótesis indica la relación completa - por ejemplo H10dmuestra un efecto total en la percepción de la calidad de servicio. 


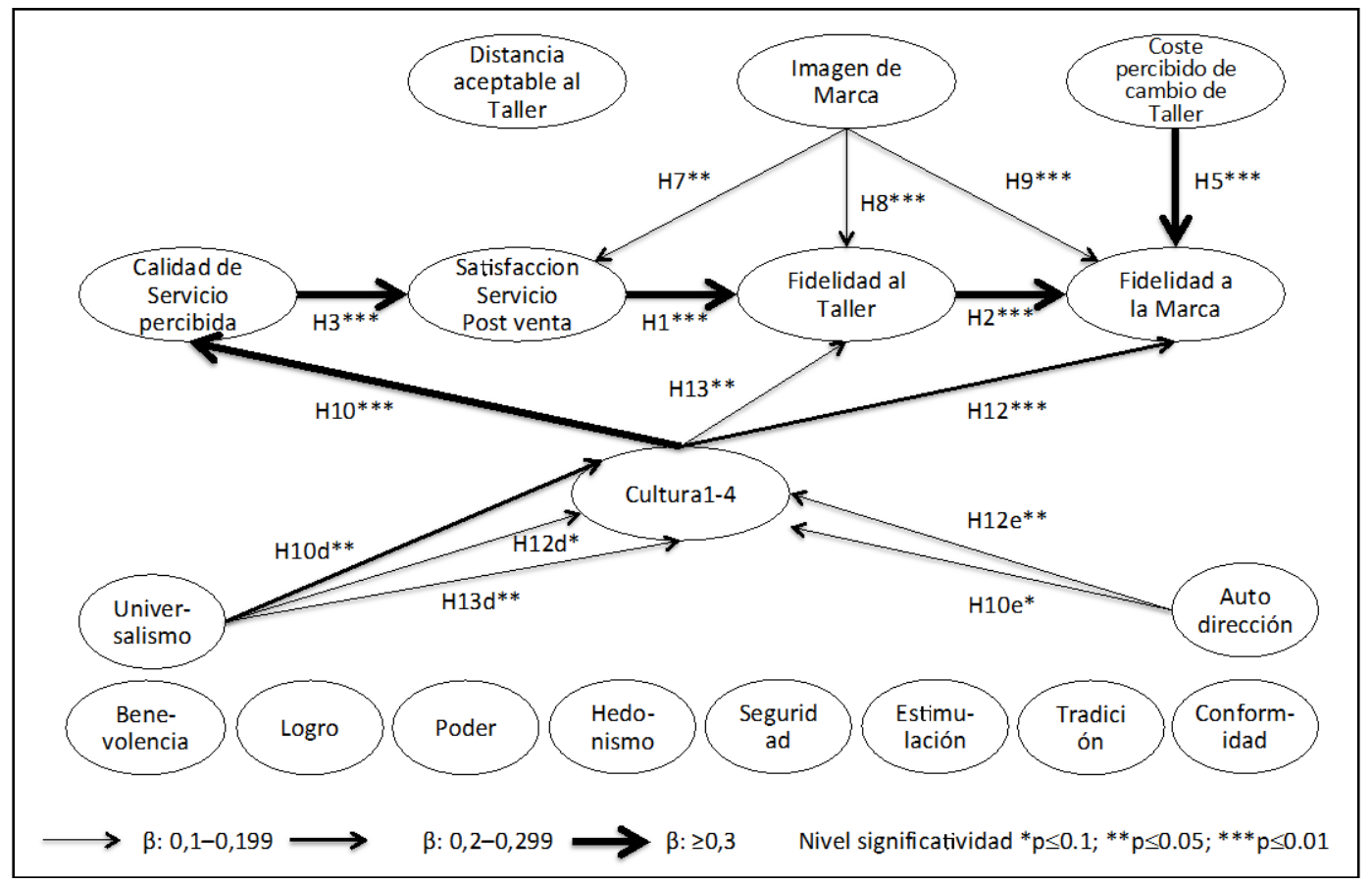

Figura 5. Fuerza de Empíricamente Confirmado Hipótesis del Modelo Path (autores)

\section{Conclusiones. Implicaciones de gestión.}

Este trabajo ofrece una guía conceptual para analizar los procesos de servicios posventa automotriz en países emergentes como China y los factores que influyen en el éxito de los mismos. Para ello se ha propuesto un modelo que relaciona la cadena de valor de éxito en los procesos postventa del automóvil (en su segmento de lujo o premium) y cómo influyen en la misma los valores culturales de la población a la que pertenecen los individuos consumidores del mismo.

Como elemento fundamental se ha adoptado y justificado la fidelidad a la marca como un indicador de éxito en esa cadena de valor postventa del sector automóvil. Por otra parte, este artículo presenta un enfoque innovador que cubre un hueco importante en la investigación sobre los procesos post venta del automóvil al incorporar la influencia de los valores culturales locales en estos procesos y como estos (individual y globalmente) afectan a la conducta del consumidor final

Los resultados muestran que la fidelidad a la marca se explica bien (a un nivel del 64,8\%) por medio de las variables exógenas del modelo propuesto. La propuesta de la cadena de valor de éxito en los procesos postventa del automóvil (en países emergentes) se puede confirmar como válida para China. Así, la calidad percibida de servicio post venta influye significativamente en la satisfacción del servicio post-venta, que en sí misma es un predictor fuerte de la fidelidad al taller. Por otra parte, los consumidores-clientes fieles al taller postventa tienden a ser, además, de forma significativa fieles a la marca objeto de análisis. Esto se fomenta a través de la percepción de los costes de cambio de taller. Es de subrayar que la imagen de marca resulta un elemento clave y deseable ya que afecta simultáneamente a las variables satisfacción de servicio y lealtad a la marca.

Por último, debe apuntarse que la influencia de los valores culturales locales o nacionales se ha verificado empíricamente con la única excepción de la satisfacción del servicio post-venta. Además, este trabajo muestra precisamente que valores culturales a nivel individual son realmente causales. Entre estos, el universalismo (e.g., la comprensión, apreciación, tolerancia y protección hacia el bienestar de todas las personas y la naturaleza) y la auto afirmación (e.g., el pensamiento independiente y elección propia de la acción, creando y explorando) contribuyen positivamente a la percepción de la calidad del servicio y los comportamientos de fidelidad.

Estas conclusiones tienen implicaciones en la gestión y enfoque de la mercadotecnia de servicios en el sector del automóvil.

En primer lugar se subraya la importancia del proceso post venta y de la mercadotecnia de este servicio en si ya que son relevantes en la contribución a la lealtad de marca, a la imagen de marca y a la repetición del 
comprador. Especialmente en países emergentes que, es donde precisamente se concentran los mercados sujetos a mayores cuotas de crecimiento.

Los gestores en estos países de las macas premium deberán prestar especial atención a la satisfacción del servicio post-venta ya que aparece como un predictor fuerte de la fidelidad al taller. Y como se ha demostrado, los consumidores-clientes fieles al taller postventa tienden a ser fieles a la marca objeto de análisis. De ahí que deberán monitorizar variables como la satisfacción sobre los servicios del taller y la propensión al cambio de taller y relacinarlos con la percepción de imagen de marca.

Por otra parte, desde una perspectiva global, los fabricantes deberán tratar de segmentar sus mercados y cuidar la postventa aplicando diferencias culturales para su comunicación y gestión de clientes. Así hemos podido comprobar como valores de autoafirmación o universalismo aparecen como cruciales para el consumidor. Con relación al último los valores relacionados con el medio ambiente resultan de interés para el consumidor en contra de lo que se pudiera pensar.

\section{Limitaciones de este estudio}

Este trabajo de investigación se basa en fundamentos teóricos y conceptuales holísticos, y los resultados se evalúan con cierta cautela. Sin embargo, como en todo trabajo empírico, existen algunas limitaciones. En primer lugar, el mercado objeto del trabajo, el segmento premium de automóvil, supone en si cierta limitación en cuanto a su generalización en un mercado completo. Las investigaciones futuras deben abordar otros segmentos más amplios como el de bajo coste. En segundo lugar, aunque la teoría de los valores culturales individuales de Schwartz's presenta un enfoque interesante, no debe olvidarse que existen otros enfoques. Recientemente se ha presentado un enfoque mas sofisticado con 19 valores culturales (Cieciuch et al., 2014a, 2014b; Schwartz et al., 2012, Schwartz, 2011b), que podrían enriquecer el cuerpo académico de conocimiento con perspectivas adicionales. Sin embargo, si el modelo de investigación (básico) de factores no-culturales es más complejo o bien los recursos son limitados, el enfoque utilizado de los 10 valores es mas recomendable. Finalmente, este trabajo muestra que los efectos de los valores culturales influyen sobre aspectos importantes del comportamiento del consumidor, pero no se han considerado los efectos de moderación. Empero, estos pueden ser cruciales, como por ejemplo las diferencias de género. Algunos análisis multi-grupo no divulgados indican la existencia de estos efectos de moderación, por ello las investigaciones futuras debe centrarse en ellos $y$, especialmente, con relación a la causalidad de los valores de nivel individual.

\section{Referencias}

ASUGMAN, G., JOHNSON, J. L. \& MC CULLOUGH, J. (1997). "The Role of After-Sales Service in International Marketing". Journal of International Marketing, 5 (4), pp. 11-28.

BECKER, J.-M., KLEIN, K. \& WETZELS, M. (2012). "Hierarchical Latent Variable Models in PLS-SEM: Guidelines for Using Reflective-Formative Type Models". Long Range Planning, 45, pp. 359-394.

BEI, L.-T. \& CHIAO, Y.-C. (2001). "An Integrated Model for the Effects of Perceived Product, Perceived Service Quality, and Perceived Price Fairness on Consumer Satisfaction and Loyalty". Journal of Consumer Satisfaction Dissatisfaction and Complaining Behavior, 14, pp. 125-140.

BLOEMER, J. M. M. \& PAUWELS, K. H. (1998). "Explaining brand loyalty, dealer sales loyalty and dealer after-sales loyalty: The influence of satisfaction with the car, satisfaction with the sales service and satisfaction with the after-sales service". Journal of Consumer Satisfaction Dissatisfaction and Complaining Behaviour, 11, pp. 78-90.

BLOEMER, J. M. M. \& LEMMINK (1992). "The Importance of Customer Satisfaction in Explaining Brand and Dealer Loyalty", Journal of Marketing Management, 8 (4), pp. 351-364.

CAAM - China Association of Automobile Manufacturers (n. a.) (2013). "The passenger cars market share of Chinese brand continued to decline", available online at http://www.caam.org.cn/AutomotivesStatistics/20131014/1705103416.ht $\mathrm{ml}$, accessed 14/10/2013.

CAVALIERI, S., GAIARDELLI, P. \& IERA $\neg$ CE, S. (2007). "Aligning strategic profiles with operational metrics in after-sales service". International Journal of Productivity and Performance Management, 56 (5/6), pp. 436-455.

CIECIUCH, J. \& DAVIDOV, E. (2012). "A comparison of the invariance properties of the PVQ-40 and the $P V Q-21$ to measure human values across German and Polish Samples". Survey Research Methods, 6 (1), pp. 37-48. 
CUI, G. \& LIU, Q. (2001). "Emerging market segments in a transitional economy: a study of urban consumers in China". Journal of International Marketing, 9 (1), pp. 84-106.

DAVIDOV, E., SCHMIDT, P. \& SCHWARᄀTZ, S. H. (2008). "Bringing values back in - The adequacy of the European Social Survey to measure values in 20 countries". Public Opinion Quarterly, 72 (3), pp. 420-445.

DENG, Z., LU, Y., WEI, K. K. \& ZHANG, J. (2010). “Understanding customer satisfaction and loyalty: An empirical study of mobile instant messages in China". International Journal of Information Management, 30, pp. 289-300.

DE MOOIJ, M. (2014). Global Marketing and Advertising: Understanding Cultural Paradoxes, 4. ed., Thousand Oaks et al.: Sage Publications.

Devaraj, S., Matta, K. F. \& Conlon, E. (2001). "Product And Service Quality: The Antecedents Of Customer Loyalty In The Automotive Industry". Production and Operations Management, 10 (4), pp. 424-439.

DIAMANTOPOULOS, A., WINKELHOFER, H. M. (2001). "Index Construction with Formative Indicators: An Alternative to Scale Development, Journal of Marketing Research, 38 (2), pp. 269-277.

DIEZ, W. (2009). Automobil-Marketing - Navigationssysteme für neue Absatzstrategien, E-Book, 5. ed., München: mi-Wir?tschafts $\neg$ buch/FinanzBuch.

DIEZ, W., REINDL, S. (2005). „Das Automobil aus verkehrs- und politischer Sicht“. In: Diez, W. et al. (Eds.), Grundlagen der Automobilwirtschaft, 4. ed., pp. 21-69, München: Auto Business Verlag.

EMRICH, C. (2014). Interkulturelles Marketing-Management - Erfolgsstrategien, Konzepte, Analysen, 3. ed., Wiesbaden: Springer/Gabler.

EMRICH, C. (2007). Interkulturelles Marketing-Management - Erfolgsstrategien, Konzepte, Analysen, 2. ed., Wiesbaden: Deutscher Universitäts-Verlag/ GWV Fachverlage.

ENGELEN, A. \& BRETTEL, M. (2011). "A cross-cultural perspective of marketing departments' influence tactics". Journal of International Marketing, 19 (2), pp. 73-94.

ESCH, F.-R. (2010). Strategie und Technik der Marken-Führung, 6. ed., München: Verlag Franz Vahlen.
ESCH, F.-R. \& ISENBERG, M. (2013). "Markenidentität und Markenpositionierung festlegen", in: Esch, F.-R. (Eds.), Strategie und Technik des Automobilmarketing, pp. 35-57, Wiesbaden: Springer/Gabler.

FRANK, B., ABULAITI, G., HERBAS TORRICO, B. \& ENKAWA, T. (2013). "How do Asia's two most important consumer markets differ? Japanese-Chinese differences in customer satisfaction and its formation". Journal of Business Research, 66 (12), pp. 2397-2405.

GALLAGHER, T., MITCHKE, M. D. \& ROᄀGERS, M. C. (2005). "Profiting from spare parts", McKinsey Quarterly, 2, pp. 1-4.

GEBAUER, H., REN, G.-J., VALTAKOSKI, A. \& REYNOSO, J. (2012). "Service-driven manufacturing: provision, evolution and financial impact of services in industrial firms". Journal of Service Manage ment, 23 (1), pp. 120-136.

GOFFIN, K. (1999). "Customer support - A cross-industry study of distribution channels and strategies". International Journal of Physical Distribution and Logistics Management, 29 (6), pp. 374-397.

GOFFIN, K. \& NEW, C. (2001). "Customer support and new product development - An exploratory study". International Journal of Operations \& Production Management 21 (3), pp. 275-301.

GONG, W. (2003). "Chinese consumer behavior: a cultural framework and implications". Journal of American Academy of Business, 3 (1/2), pp. 373-380.

GRÖNROOS, C. (1984). "A Service Quality Model and its Marketing Implications". European Journal of Marketing, 18 (4), pp. 36-44.

GUDERGAN, S. (2010). "Adoption of technologies in industrial after-sales services across cultures". International Journal of Services Technology and Management, 14 (2/3), pp. 250-258

HAIR, J. F. JR., SARSTEDT, M., HOPKINS, L. \& KUPPELWIESER, V. G. (2014a). "Partial least squares structural equation modeling (PLS-SEM) - An emerging tool in business research". European Business Review, 26 (2), pp. 106-121.

HAIR, J. F. JR., HULT, G. T. M./RINGLE, C. M./ SARSTEDT, M. (2014b). A Primer on Partial Least Squares Structural Equation Modeling (PLS-SEM), Los Angeles et al.: Sage Publications. 
HOFSTEDE, G. (1997). Lokales Denken, globales Handeln: Kulturen, Zusammenarbeit und Management, München: Deutscher Taschenbuch Verlag.

HUBER, F. \& HERRMANN, A. (2001). "A $\urcorner$ chieving brand and dealer loyalty: the case of the automotive industry". The International Review of Retail, Distribution and Consumer Research, 11 (2), pp. 97-122.

HULL, D. \& COX, J. (1994). "The field service function in the electronics industry: Providing a link between customers and production/marketing", International Journal of Production Economics, 37 (1), pp. 115126.

HÄTTICH, H. (2009). Markenloyalität im Aftersales-Marketing - Konzept zur Erhöhung der Markenloyalität in der deutschen Automobilbranche durch Optimierung eines herstellerinitiierten Aftersales-Marketing, München \& Mering: Rainer Hampp Verlag.

HÜNECKE, P. (2012). Einfluss von After-Sales-Service Determinanten auf die Marken-loyalität im Premiumautomobilsektor - eine empirische Analyse in drei Märkten, Göttingen: Cuvillier Verlag.

JÖNKE, R. (2012). "Managing after-sales services: strategies and interfirm relationships", available online at http://dx.doi.org/ 10.3929/ethz-a-007307425, accessed 17/5/2013.

KÖLL, E. (2009). "JWT China: Advertising for the New Chinese Consumer". HBS Case, 9-809-079, pp. 1-26.

KNÖRLE, C. (2011). Markenloyalität in China - Kulturelle und markenbeziehungstheoretische Determinanten, Berlin: Logos.

LAM, D. (2007). "Cultural Influence on Proneness to Brand Loyalty". Journal of International Consumer Marketing, 19 (3), pp. 7-21.

LAI, F., GRIFFIN, M. \& BABIN, B. J. (2009). "How quality, value, image, and satisfaction create loyalty at a Chinese telecom". Journal of Business Research, 62, pp. 980-986.

LERCHENMÜLLER, M. (2007). „Die Distributionspolitik im After Sales Service“, In: Pepels, W. (Eds.), After Sales Service - Geschäftsbeziehungen profitable gestalten, 2. ed., pp. 157-188, Düsseldorf: Symposium Publishing.

LI, D., AN, S. \& YANG, K. (2008). "Exploring Chinese consumer repurchasing intention for services: an empirical investigation". Journal of Consumer Behaviour, 7 (6), pp. 448-460.

LÖFFLER, M. \& DECKER, R. (2012). "Service Satisfaction With Premium Durables: A Cross-Cultural Investigation". In: Diamantopoulos, A. et al. (Eds.), Quantitative Marketing and Marketing Management - Marketing Models and Methods in Theory and Practice, pp. 401-422, Wiesbaden: Gabler.

MEFFERT, H., BURRMANN, C. \& BECKER, C. (2010). Internationales Marketing-Management - Ein markenorientierter Ansatz, 4. ed., Stuttgart: Kohlhammer.

MEFFERT, H. \& BRUHN, M. (2009). Dienstleistungsmarketing - Grundlagen - Konzepte - Methoden, Wiesbaden: Gabler.

MÜLLER, S. \& GELBRICH, K. (2004). Interkulturelles Marketing, München: Verlag Franz Vahlen.

OGBA, I.-E. \& TAN, Z. (2009). "Exploring the impact of brand image on customer loyalty and commitment in China". Journal of Technology Management in China, 4 (2), pp. 132-144.

OSGOOD, C. E. (1951). "Culture: its empirical and non-empirical character". Southwestern Journal of Anthropology, 7 (2), pp. 202-214.

PETER, S. I. (1998). „Beziehungsmanage 7 ment Kundenbindung als Marketingziel“. Absatzwirtschaft, 7, pp. 74-81.

REICHHUBER, A. W. (2010). Strategie und Struktur in der Automobilindustrie - Strategische und organisatorische Programme zur Handhabung automobilwirtschaftlicher Herausforderungen, Wiesbaden: Gabler.

REIMANN, M., LÜNEMANN, U. F. \& CHASE, R. B. (2008). "Uncertainty Avoidance as a Moderator of the Relationship between Perceived Service Quality and Customer Satisfaction". Journal of Service Research, 11 (1), pp. 63-73.

SACCANI, N., SONGINI, L. \& GAIARDEL $\neg$ LI, P. (2006). "The role and performance measurement of after-sales in the durable consumer goods industries: an empirical study". International Journal of Productivity and Performance Management, 55 (3/4), pp. 259-283.

SCHWARTZ, S. H. (2011b). "Studying Val-ues: Personal Adventure, Future Directions". Journal of Cross-Cultural Psychology, 42 (2), pp. 307-319. 
SCHWARTZ, S. H. (2006). "A Theory of Cultural Value Orientations: Explication and Applications". Comparative Sociology, 5 (2/3), pp. 137-182.

SCHWARTZ, S. H. (2003). "A Proposal for Measuring Value Orientations Across Nations, The European Social Survey (ESS)". available online at http:// www.europeansoc ialsurvey.org/docs/methodologyy/core_ess_questionnaire /ESS_core_questionnaire_human_values.pdf, accessed 01/10/2014, pp. 259-319.

SCHWARTZ, S. H. (1994). "Are There Universal Aspects in the Structure and Contents of Human Values?". Journal of Social Issues, 50 (4), pp. 19-45.

SCHWARTZ, S. H. (1992). "Universals in the content and structure of values: Theoretical advances and empirical tests in 20 countries". Advances in Experimental Social Psychology, 25 (1), pp. 1-65.

SCHWARTZ, S. H. \& BOEHNKE, K. (2004). "Evaluating the Structure of Human Values with Confirmatory Factor Analysis", Journal of Research in Personality, 38 (3), pp. 230-255.

SCHWARTZ, S. H., CIECIUCH, J., VECCHIONE, M., DAVIDOV, E., FISCHER, R., BEIERLEIN, C., RAMOS, A., VERKASALO, M., LÖNNQVIST, J.-E., DEMIRUTKU, K., DIRILEN-GUMUS, O. \& KONTY, M. (2012). "Refining the Theory of Basic Individual Values". Journal of Personality and Social Psychology, 103 (4), pp. 663-688.

SIVADAS, E. \& BACKER-PREWITT, J. L. (2000). "An examination of the relationship between service quality, customer satisfaction, and store loyalty". International Journal of Retail and Distribution Management, 28 (2), pp. 73-82.

SHA, S., HUANG, T. \& GABARDI, E. (2013). "Upward Mobility: The future of China's Premium Car Market", McKinsey Automotive \& Assembly Practice in Greater China, available online at http://www.mckin seychina.com/2013/03/07/upw ard-mobility-the-future-of-chinas-premium-car-market/, accessed 10/6/2013.

SHUQIN, W. \& GANG, L. (2012). "An Empirical Study of After-sales Service Relationship in China's Auto Industry". Atlantic Press, Proceedings of 2012 International Conference on Mechanical Engineering and Material Science (MEMS), pp. 175-178.

SRNKA, K. (2005). "marketing.ethik.\&kultur". München \& Mering: Rainer Hampp Verlag.
THOMPSON, F. M., NEWMAN, A. \& LIU, M. (2014). "The moderating effect of individual level collectivist values on brand loyalty". Journal of Business Research, 67, pp. 2437-2446.

TRIANDIS, H. C. (1994). Culture and Social Behaviour, New York et al.: McGraw Hill.

TU, Y.-T., LIU, W.-C. \& CHANG, Y.-Y. (2014). "Customer Commitment as a Mediating Variable between Corporate Brand Image and Customer Loyalty". Journal of Education and Vocational Research, 5 (1), pp. 17-27.

VERHOEF, P. C., LANGERAK, F. \& DONKERS, B. (2007). "Understanding brand and dealer retention in the new car market: The moderating role of brand tier". Journal of Retailing, 83 (1), pp. 97-113.

VDA - Verband der Automobilindustrie e. V. (n. a.) (2015). "Jahresbericht 2015", available online at https://www.vda.de/de/s ervices/Publikationen/ jahresbericht-2015.ht ml, accessed 26/8/2015.

VAN BIRGELEN, M., DE RUYTER, K., DE JONG, A. \& WETZELS, M. (2002). "Customer evaluations of after-sales service contact modes: an empirical analysis of national culture's con $\urcorner$ sequences". International Journal of Research in Marketing, 19 (1), pp. 43-64.

WANG, C.-Y. (2010). "Service Quality, Perceived Value, Corporate Image, and Customer Loyalty in the Context of Varying Levels of Switching Costs". Psychology \& Marketing, 27 (3), pp. 252-262.

WANG, Q. (2011). "Development and trends of China's automobile market: evidence from urban household ownership of cars, bicycles, motorcycles and motorbikes". International Journal of Automotive Technology and Management, 11 (2), pp. 99-113.

WANG, A., LIAO, W., HEIN, A.-P. (2012). "Bigger, better, broader: A perspective on China's auto market in 2020". In: McKinsey\&Company (Eds.), Automotive \& Assembly Practice, available online at http://c si.mckinsey.com/knowledge_by_regi on/asia/china/chinas_a uto_market_in_2020, accessed 15/10/2013.

WETZELS, M., ODERKERKEN-SCHRÖᄀDER, G. \& VAN OPPEN, C. (2009). "Using PLS Path Modeling For Assessing Hierachical Construct Models: Guidelines And Empirical Illustration". MIS Quarterly, 33 (1), pp. 177-195.

WISE, R. \& BAUMGARTNER, P. (1999). "Go down- 
stream - The New Profit Imperative in Manufacturing", Harvard Business Review, 77 (5), pp. 133-141.

XIAO, G. \& KIM, J. (2009). "The Investigation of Chinese Consumer Values, Consumption Values, Life Satisfaction, and Consumption Behaviors". Psychology \& Marketing, 26 (7), pp. 610-624.

XIN-AN, Z., GRIGORIOU, N. \& LY, L. (2008). "The myth of China as a single market - The influence of personal value differences on buying decisions". International Journal of Market Research, 50 (3), pp. 377-402.

YIM, C. K., TSE, D. K. \& CHAN, K. W. (2008). "Strengthening Customer Loyalty Through Intimacy and Passion: Roles of Customer-Firm Affection and
Customer-Staff Relationships in Services", Journal of Marketing Research, 45 (6), pp. 741-756.

YOO, B. (2009). "Cross-national invariance of the effect of personal collectivistic orientation on brand loyalty and equity - The United States versus South Korean consumers", Asia Pacific Journal of Marketing and Logistics, 21 (1), pp. 41-57.

ZHANG, J., BEATTY, S. E. \& WALSH, G. (2008). "Review and future directions of cross-cultural consumer services research", Journal of Business Research, 61, pp. 211-224.

\section{Apéndice}

Anexo 1: Evaluación de modelos de medición reflexivos

\begin{tabular}{|c|c|c|c|c|c|c|c|}
\hline \multicolumn{8}{|c|}{ Evaluación de modelos de medición reflexivos } \\
\hline \multicolumn{2}{|l|}{ Criterios } & $\begin{array}{c}\text { Fiabilidad } \\
\text { compuesta }\end{array}$ & $\begin{array}{l}\text { Fiabilidad del } \\
\text { indicador }\end{array}$ & $\begin{array}{l}\text { T-estadísti- } \\
\text { cas } \\
\text { (Significado) }\end{array}$ & AVE & $\begin{array}{l}\text { No Cruz } \\
\text { cargas }\end{array}$ & $\begin{array}{l}\text { Criterio } \\
\text { de For- } \\
\text { nell-Larck- } \\
\quad \text { er }\end{array}$ \\
\hline \multicolumn{2}{|l|}{ Nivel del requisito } & $c=0.7-0.9$ & $>0.708$ & $>1,96$ & $>0.5$ & $>0.1$ & $\square$ AVE > $\square$ \\
\hline Construir & Artículo & & & & & & \\
\hline \multirow{2}{*}{$\begin{array}{l}\text { Satisfacción servi- } \\
\text { cio post-venta- }\end{array}$} & ASSS_1_v_26 & \multirow[t]{2}{*}{0,900} & 0.898 & $63.657^{* * *}$ & \multirow[t]{2}{*}{0.819} & & \\
\hline & ASSS_2_v_27 & & 0.912 & $88.632^{* * *}$ & & & \\
\hline \multirow{2}{*}{$\begin{array}{l}\text { Fidelización al tall- } \\
\text { er }\end{array}$} & WL_1_v_54 & \multirow[t]{2}{*}{0.890} & 0.903 & $60.896^{* * *}$ & \multirow[t]{2}{*}{0.802} & & \\
\hline & WL_2_v_55 & & 0,888 & $51.080 * * *$ & & & \\
\hline \multirow{3}{*}{$\begin{array}{l}\text { Fidelidad a la mar- } \\
\text { ca }\end{array}$} & BL_1_v_59 & \multirow[t]{3}{*}{0.875} & 0.846 & $34.958^{* * *}$ & \multirow[t]{3}{*}{0.701} & & \\
\hline & BL_2_v_60 & & 0.822 & $34.678^{* * *}$ & & & \\
\hline & BL_3_v_61 & & 0.844 & $38.651^{* * *}$ & & & \\
\hline \multirow[t]{3}{*}{ Imagen de marca } & BI_1_v_64 & \multirow[t]{3}{*}{0.856} & 0.869 & $52.519^{* * *}$ & \multirow[t]{3}{*}{0.664} & & \\
\hline & BI_2_v_65 & & 0.812 & $29.197^{* * *}$ & & & \\
\hline & BI_3_v_66 & & 0.761 & $27.889^{* * *}$ & & & \\
\hline \multirow{2}{*}{$\begin{array}{l}\text { Percepción costes } \\
\text { cambio de Taller } \\
\text { post venta }\end{array}$} & PWSC_1_v_44 & \multirow[t]{2}{*}{0.855} & 0.887 & $51.517^{* * *}$ & \multirow[t]{2}{*}{0.747} & & \\
\hline & PWSC_2_v_45 & & 0.841 & $24.450 * * *$ & & & \\
\hline
\end{tabular}




\begin{tabular}{|c|c|c|c|c|c|c|c|}
\hline \multirow[t]{2}{*}{ Autoafirmacion } & SED_1_v_78 & \multirow[t]{2}{*}{0.866} & 0.870 & $46.293^{* * *}$ & \multirow[t]{2}{*}{0.763} & & \\
\hline & SED_2_v_88 & & 0.877 & $47.218^{* * *}$ & & & \\
\hline \multirow[t]{2}{*}{ Universalismo } & UNI_1_v_80 & \multirow[t]{2}{*}{0.881} & 0884 & $55.554^{* * *}$ & \multirow[t]{2}{*}{0.788} & & \\
\hline & UNI_2_v_85 & & 0.891 & $56.416^{* * *}$ & & & \\
\hline \multirow[t]{2}{*}{ Benevolencia } & BEN_1_v_152 & \multirow[t]{2}{*}{0.841} & 0.880 & $46.416^{* * *}$ & \multirow[t]{2}{*}{0.726} & & \\
\hline & BEN_2_v_158 & & 0.823 & $24.803^{* * *}$ & & & \\
\hline \multirow[t]{2}{*}{ Tradición } & TRA_1_v_86 & \multirow[t]{2}{*}{0.837} & 0.885 & $36.801^{* * *}$ & \multirow[t]{2}{*}{0.720} & & \\
\hline & TRA_2_v_160 & & 0.811 & $18.445^{* * *}$ & & & \\
\hline \multirow[t]{2}{*}{ Conformidad } & CON_1_v_84 & \multirow[t]{2}{*}{0.835} & 0.821 & 22.139 *** & \multirow[t]{2}{*}{0,716} & & \\
\hline & CON_2_v_156 & & 0.871 & $42.613^{* * *}$ & & & \\
\hline \multirow[t]{2}{*}{ Seguridad } & SEC_1_v_82 & \multirow[t]{2}{*}{0.812} & 0.826 & $21.312^{* * *}$ & \multirow[t]{2}{*}{0.683} & & \\
\hline & SEC_2_v_154 & & 0.827 & 25.256 *** & & & \\
\hline \multirow[t]{2}{*}{ Energía } & POW_1_v_79 & \multirow[t]{2}{*}{0.839} & 0867 & $31.677^{* * *}$ & \multirow[t]{2}{*}{0.723} & & \\
\hline & POW_2_v_157 & & 0.834 & 23.423 *** & & & \\
\hline \multirow[t]{2}{*}{ Logro } & ACH_1_v_81 & \multirow[t]{2}{*}{0872} & 0.885 & $51.561^{* \star *}$ & \multirow[t]{2}{*}{0.773} & & \\
\hline & ACH_2_v_153 & & 0.874 & $42.773^{* * *}$ & & & \\
\hline \multirow[t]{2}{*}{ Hedonismo } & HED_1_v_87 & \multirow[t]{2}{*}{0.876} & 0.878 & $38.782 * * *$ & \multirow[t]{2}{*}{0,779} & & \\
\hline & HED_2_v_161 & & 0.887 & $43.781^{* * *}$ & & & \\
\hline \multirow[t]{2}{*}{ Estimulación } & STI_1_v_83 & \multirow[t]{2}{*}{0.865} & 0.905 & $66.257^{* * *}$ & \multirow[t]{2}{*}{0.762} & & \\
\hline & STI_2_V_155 & & 0.840 & $28.529 * * *$ & & & \\
\hline
\end{tabular}


Anexo 2: Evaluación de los modelos de medición formativos

\begin{tabular}{|c|c|c|c|c|}
\hline \multicolumn{5}{|c|}{ Evaluación formativa modelos de medición } \\
\hline \multicolumn{2}{|l|}{ Criterios } & VIF & Peso externo & valor de $t$ \\
\hline \multicolumn{2}{|l|}{ Nivel del requisito } & $<3,3$ & $\mathrm{n} / \mathrm{a}$ & 1,96 \\
\hline Constructor & Indicador de & & & \\
\hline \multirow[t]{9}{*}{ Calidad de servicio percibida } & PSQ_1_v15 & 1.382 & $0.142^{*}$ * & 2.032 \\
\hline & PSQ_2_v13 & 1.583 & $0.280^{* * *}$ & 3947 \\
\hline & PSQ_3_v_7 & 1.565 & $0.108^{*}$ & 1.793 \\
\hline & PSQ_4_v_8 & 1.565 & $0.199^{* * *}$ & 2.963 \\
\hline & PSQ_5_v_9 & 1.914 & $0.206^{* * *}$ & 2.766 \\
\hline & PSQ_6_v_10 & 2.058 & $0.147^{*}$ * & 2.059 \\
\hline & PSQ_7_v_185 & 1.875 & -0.047 & 0.703 \\
\hline & PSQ_8_v_11 & 1.500 & $0.234^{* * *}$ & 3.481 \\
\hline & PSQ_9_v_14 & 1.718 & $0.184^{* * *}$ & 2.773 \\
\hline
\end{tabular}

Anexo 3: Evaluación del modelo estructural

\begin{tabular}{|c|c|c|c|c|c|}
\hline \multicolumn{6}{|c|}{ Evaluación del modelo estructural } \\
\hline Criterios & & $\mathbf{R}^{2}$ & $f^{2}$ & $\mathbf{Q}^{2}$ & $q^{2}$ \\
\hline Construir $\square$ & Nivel del requisito & $\begin{array}{c}\text { Mínimo > } 0.25 \\
>0,5 ;>0.75\end{array}$ & $\begin{array}{l}\text { Mínimo > 0,02 } \\
>0,15 ;>0,35\end{array}$ & $>0$ & $\begin{array}{l}\text { Mínimo }>0,02 \\
>0,15 ;>0,35\end{array}$ \\
\hline \multicolumn{2}{|c|}{ Calidad de servicio percibida } & 0.361 & $\square$ ASSS: 0.643 & $\mathrm{n} / \mathrm{a}$ & $\mathrm{n} / \mathrm{a}$ \\
\hline \multicolumn{2}{|c|}{ Satisfacción servicio post-venta- } & 0.633 & $\square \mathrm{WL}: 0.552$ & 0.491 & $\square$ WL: 0.059 \\
\hline \multicolumn{2}{|c|}{ Fidelización de taller } & 0.643 & $\square$ BL: 0.180 & 0.492 & $\square$ BL: 0,033 \\
\hline \multicolumn{2}{|c|}{ Fidelidad a la marca } & 0.648 & & 0.427 & \\
\hline \multicolumn{2}{|c|}{ Imagen de marca } & & $\begin{array}{l}\square \text { ASSS: } 0,033 \\
\square \text { WL: } 0.034 \\
\square \text { BL: } 0.034\end{array}$ & & $\begin{array}{l}\square \text { ASSS: } 0,051 \\
\square \text { WL:-0.003 } \\
\square \text { BL: } 0.024\end{array}$ \\
\hline \multicolumn{2}{|c|}{ Percepción costes cambio de Taller post venta } & & $\begin{array}{l}\square \text { WL: } 0.001 \\
\square \text { BL: } 0.210\end{array}$ & & $\begin{array}{l}\square \text { WL: }-0,020 \\
\square \text { BL: } 0.068\end{array}$ \\
\hline \multicolumn{2}{|c|}{ Distancia aceptable al taller } & & $\begin{array}{l}\square \text { ASSS: } 0.000 \\
\square \text { WL: } 0.003\end{array}$ & & $\begin{array}{l}\square \text { ASSS: }-0,020 \\
\square \text { WL: } 0.031\end{array}$ \\
\hline
\end{tabular}


Anexo 4: Resumen de la validación de hipótesis

\begin{tabular}{|c|c|c|c|c|c|}
\hline \multicolumn{6}{|c|}{ Resumen de la prueba de hipótesis } \\
\hline \multicolumn{2}{|c|}{$\begin{array}{l}\text { Criterios } \\
\text { Nivel del requisito }\end{array}$} & \multirow[t]{2}{*}{$\square$} & \multirow[t]{2}{*}{$\begin{array}{l}\text { valor de t } \\
1.65 \square\end{array}$} & \multirow[t]{2}{*}{$\mathrm{f}^{2}$ pequeño } & \multirow{2}{*}{$\begin{array}{l}\text { Todos los } \\
\text { criterios } \\
\text { cumplido } \\
\text { Resultado }\end{array}$} \\
\hline Hipótesis & Hipótesis & & & & \\
\hline H1 & $\begin{array}{l}\text { Cuanto mayor sea la satisfacción de servicio post venta, mayor } \\
\text { será la fidelidad de taller. }\end{array}$ & $0.578^{* * *}$ & 7.733 & Grandes & Aceptado \\
\hline $\mathrm{H} 1$ & $\begin{array}{l}\text { Cuanto mayor sea la satisfacción de servicio post venta, mayor } \\
\text { será la fidelidad de taller. }\end{array}$ & $0.578^{* * *}$ & 7.733 & Grandes & Aceptado \\
\hline $\mathrm{H} 2$ & $\begin{array}{l}\text { Cuanto mayor sea la fidelidad de taller, cuanto mayor sea la fidel- } \\
\text { idad a la marca. }\end{array}$ & $0.331^{* * *}$ & 5.453 & Medio & Aceptado \\
\hline H3 & $\begin{array}{l}\text { Cuanto mayor sea la calidad del servicio percibida, mayor será la } \\
\text { satisfacción de servicio post venta. }\end{array}$ & $0.673^{* * *}$ & 10.723 & Grandes & Aceptado \\
\hline H4 & $\begin{array}{l}\text { Cuanto mayor sea la percepción de costes de cambio de Taller } \\
\text { post venta mayor sea la fidelidad al taller. }\end{array}$ & 0.022 & 0.448 & $x$ & Rechazado \\
\hline H5 & $\begin{array}{l}\text { Cuanto mayor sea la percepción de costes de cambio de Taller } \\
\text { post venta mayor sea la fidelidad a la marca. }\end{array}$ & $0.305^{* * *}$ & 5.720 & Medio & Aceptado \\
\hline H6 & $\begin{array}{l}\text { Existe una relación entre la distancia aceptable al taller y la fideli- } \\
\text { dad al taller y la satisfacción del servicio post venta. }\end{array}$ & $\begin{array}{l}0.003 \\
0.031\end{array}$ & $\begin{array}{l}0.143 \\
1.032\end{array}$ & $\begin{array}{l}x \\
x\end{array}$ & Rechazado \\
\hline $\mathrm{H} 7$ & $\begin{array}{l}\text { Cuanto mayor sea la imagen de marca, mayor será la satisfacción } \\
\text { del servicio post venta. }\end{array}$ & $0.162^{* *}$ & 2.523 & Pequeño & Aceptado \\
\hline H8 & $\begin{array}{l}\text { Cuanto mayor sea la imagen de marca, mayor será la fidelidad } \\
\text { de taller. }\end{array}$ & $0.165^{* * *}$ & 2.629 & Pequeño & Aceptado \\
\hline H9 & $\begin{array}{l}\text { Cuanto mayor sea la imagen de marca, mayor será la fidelidad a } \\
\text { la marca. }\end{array}$ & $0.162^{* * *}$ & 2.730 & Pequeño & Aceptado \\
\hline H10 & $\begin{array}{l}\text { La percepción de calidad del servicio está influenciada significa- } \\
\text { tivamente por los valores culturales. }\end{array}$ & $0.601^{* * *}$ & 14.982 & Grandes & Aceptado \\
\hline $\mathrm{H} 10 \mathrm{a}$ & $\begin{array}{l}\text { La Percepción de la calidad del servicio está influenciada significativa- } \\
\text { mente por conformidad. }\end{array}$ & 0.127 & 1.239 & $\mathrm{n} / \mathrm{a}$ & Rechazado \\
\hline $\mathrm{H} 10 \mathrm{~b}$ & $\begin{array}{l}\text { La Percepción de calidad del servicio está influenciada significativa- } \\
\text { mente por la tradición. }\end{array}$ & -0.045 & 0.659 & $\mathrm{n} / \mathrm{a}$ & Rechazado \\
\hline $\mathrm{H} 10 \mathrm{c}$ & $\begin{array}{l}\text { La Percepción de la calidad del servicio está influenciada significativa- } \\
\text { mente por benevolencia. }\end{array}$ & 0.035 & 0,311 & $\mathrm{n} / \mathrm{a}$ & Rechazado \\
\hline $\mathrm{H} 10 \mathrm{~d}$ & $\begin{array}{l}\text { La Percepción de la calidad del servicio está influenciada significativa- } \\
\text { mente por universalismo. }\end{array}$ & $0.205^{\star \star}$ & 2.173 & $\mathrm{n} / \mathrm{a}$ & Aceptado \\
\hline
\end{tabular}




\begin{tabular}{|c|c|c|c|c|c|}
\hline $\mathrm{H} 10 \mathrm{e}$ & $\begin{array}{l}\text { La Percepción de la calidad del servicio está influenciada significativa- } \\
\text { mente por uno mismo-dirección. }\end{array}$ & $0.187^{*}$ & 1.910 & $\mathrm{n} / \mathrm{a}$ & Aceptado \\
\hline $\mathrm{H} 10 \mathrm{f}$ & $\begin{array}{l}\text { La Percepción de la calidad del servicio está influenciada significativa- } \\
\text { mente por el estímulo. }\end{array}$ & 0.139 & 1.486 & $\mathrm{n} / \mathrm{a}$ & Rechazado \\
\hline $\mathrm{H} 10 \mathrm{~g}$ & $\begin{array}{l}\text { La Percepción de la calidad del servicio está influenciada significativa- } \\
\text { mente por hedonismo. }\end{array}$ & 0.102 & 0.962 & $\mathrm{n} / \mathrm{a}$ & Rechazado \\
\hline $\mathrm{H} 10 \mathrm{~h}$ & $\begin{array}{l}\text { La Percepción de la calidad del servicio está influenciada significativa- } \\
\text { mente por logro. }\end{array}$ & -0.135 & 1.290 & $\mathrm{n} / \mathrm{a}$ & Rechazado \\
\hline $\mathrm{H} 10 \mathrm{i}$ & $\begin{array}{l}\text { La Percepción de la calidad del servicio está influenciada significativa- } \\
\text { mente por poder. }\end{array}$ & -0.013 & 0.161 & $\mathrm{n} / \mathrm{a}$ & Rechazado \\
\hline $\mathrm{H} 10 \mathrm{k}$ & $\begin{array}{l}\text { La Percepción de la calidad del servicio está influenciada significativa- } \\
\text { mente por seguridad. }\end{array}$ & 0,114 & 1.349 & $\mathrm{n} / \mathrm{a}$ & Rechazado \\
\hline H11 & $\begin{array}{l}\text { Satisfacción del servicio post-venta está influenciada significati- } \\
\text { vamente por la cultura. }\end{array}$ & 0.010 & 0,151 & $x$ & Rechazado \\
\hline $\begin{array}{l}\mathrm{H} 11 \\
\mathrm{a}-\mathrm{k}\end{array}$ & $\begin{array}{l}\text { La Satisfacción del servicio post-venta está significativamente influen- } \\
\text { ciada por laconformidad, tradición, benevolencia, universalismo, auto- } \\
\text { dirección, estimulación, hedonismo, logro, poder o seguridad }\end{array}$ & $x$ & $x$ & $x$ & Rechazado \\
\hline $\mathrm{H} 12$ & $\begin{array}{l}\text { Fidelidad a la marca es significativamente influenciado por la cul- } \\
\text { tura. }\end{array}$ & $0.242^{* * *}$ & 4.141 & Pequeño & Aceptado \\
\hline $\mathrm{H} 12 \mathrm{a}$ & $\begin{array}{l}\text { La Fidelidad a la marca está significativamente influenciada por con- } \\
\text { formidad. }\end{array}$ & 0.029 & 0.511 & $\mathrm{n} / \mathrm{a}$ & Rechazado \\
\hline $\mathrm{H} 12 \mathrm{~b}$ & $\begin{array}{l}\text { La Fidelidad a la marca es significativamente influenciado por la } \\
\text { tradición. }\end{array}$ & 0.040 & 0.889 & $\mathrm{n} / \mathrm{a}$ & Rechazado \\
\hline $\mathrm{H} 12 \mathrm{c}$ & $\begin{array}{l}\text { La Fidelidad a la marca está significativamente influenciada por be- } \\
\text { nevolencia. }\end{array}$ & 0000 & 0.007 & $\mathrm{n} / \mathrm{a}$ & Rechazado \\
\hline $\mathrm{H} 12 \mathrm{~d}$ & $\begin{array}{l}\text { La Fidelidad a la marca es significativamente influenciado por el uni- } \\
\text { versalismo. }\end{array}$ & $0.101^{*}$ & 1.799 & $\mathrm{n} / \mathrm{a}$ & Aceptado \\
\hline $\mathrm{H} 12 \mathrm{e}$ & $\begin{array}{l}\text { La Fidelidad a la marca es influenciada significativamente por la au- } \\
\text { toafirmación. }\end{array}$ & $0.136^{* *}$ & 2.376 & $\mathrm{n} / \mathrm{a}$ & Aceptado \\
\hline $\mathrm{H} 12 \mathrm{f}$ & $\begin{array}{l}\text { La Fidelidad a la marca está influenciada significativamente por el es- } \\
\text { tímulo. }\end{array}$ & 0.059 & 1.182 & $\mathrm{n} / \mathrm{a}$ & Rechazado \\
\hline $\mathrm{H} 12 \mathrm{~g}$ & $\begin{array}{l}\text { La Fidelidad a la marca es significativamente influenciada por el he- } \\
\text { donismo. }\end{array}$ & -0.004 & 0.083 & $\mathrm{n} / \mathrm{a}$ & Rechazado \\
\hline $\mathrm{H} 12 \mathrm{~h}$ & $\begin{array}{l}\text { La Fidelidad a la marca está influenciada significativamente por el lo- } \\
\text { gro. }\end{array}$ & 0.011 & 0.193 & $\mathrm{n} / \mathrm{a}$ & Rechazado \\
\hline $\mathrm{H} 12 \mathrm{i}$ & $\begin{array}{l}\text { La Fidelidad a la marca está influenciada significativamente por el pod- } \\
\text { er. }\end{array}$ & 0.034 & 0,754 & $\mathrm{n} / \mathrm{a}$ & Rechazado \\
\hline
\end{tabular}




\begin{tabular}{|c|c|c|c|c|c|}
\hline $\mathrm{H} 12 \mathrm{k}$ & $\begin{array}{l}\text { La Fidelidad a la marca está significativamente influenciada por se- } \\
\text { guridad. }\end{array}$ & 0,064 & 1.301 & $\mathrm{n} / \mathrm{a}$ & Rechazado \\
\hline H13 & $\begin{array}{l}\text { Fidelidad de taller está influenciada significativamente por la cul- } \\
\text { tura. }\end{array}$ & $0.157^{\star *}$ & 2.427 & Pequeño & Aceptado \\
\hline $\mathrm{H} 13 \mathrm{a}$ & $\begin{array}{l}\text { La Fidelidad de taller está significativamente influenciada por confor- } \\
\text { midad. }\end{array}$ & 0.052 & 0.792 & $\mathrm{n} / \mathrm{a}$ & Rechazado \\
\hline $\mathrm{H} 13 \mathrm{~b}$ & $\begin{array}{l}\text { La Fidelidad de taller está influenciada significativamente por la } \\
\text { tradición. }\end{array}$ & -0.043 & 0.849 & $\mathrm{n} / \mathrm{a}$ & Rechazado \\
\hline H03В & $\begin{array}{l}\text { Fidelidad de taller está significativamente influenciada por benevolen- } \\
\text { cia. }\end{array}$ & 0,042 & 0.475 & $\mathrm{n} / \mathrm{a}$ & Rechazado \\
\hline $\mathrm{H} 13 \mathrm{~d}$ & $\begin{array}{l}\text { La Fidelidad de taller está significativamente influenciada por univer- } \\
\text { salismo. }\end{array}$ & $0.158^{\star *}$ & 2.152 & $\mathrm{n} / \mathrm{a}$ & Aceptado \\
\hline $\mathrm{H} 13 \mathrm{e}$ & $\begin{array}{l}\text { La Fidelidad de taller está influenciada significativamente por la au- } \\
\text { toafirmación. }\end{array}$ & 0.118 & 1.624 & $\mathrm{n} / \mathrm{a}$ & Rechazado \\
\hline $\mathrm{H} 13 \mathrm{f}$ & $\begin{array}{l}\text { La Fidelidad de taller está influenciada significativamente por el es- } \\
\text { tímulo. }\end{array}$ & 0.058 & 0.866 & $\mathrm{n} / \mathrm{a}$ & Rechazado \\
\hline $\mathrm{H} 13 \mathrm{~g}$ & $\begin{array}{l}\text { La Fidelidad de taller está significativamente influenciada por hedon- } \\
\text { ismo. }\end{array}$ & 0,042 & 0.578 & $\mathrm{n} / \mathrm{a}$ & Rechazado \\
\hline $\mathrm{H} 13 \mathrm{H}$ & La Fidelidad de taller está influenciada significativamente por el logro. & -0.035 & 0,466 & $\mathrm{n} / \mathrm{a}$ & Rechazado \\
\hline $\mathrm{H} 13 \mathrm{i}$ & La Fidelidad de taller está significativamente influenciada por poder. & -0.004 & 0.073 & $\mathrm{n} / \mathrm{a}$ & Rechazado \\
\hline $\mathrm{H} 13 \mathrm{k}$ & $\begin{array}{l}\text { La Fidelidad de taller está significativamente influenciada por seguri- } \\
\text { dad. }\end{array}$ & 0.073 & 1.144 & $\mathrm{n} / \mathrm{a}$ & Rechazado \\
\hline
\end{tabular}

